\title{
Comparison between Analog Radio-over-Fiber and Sigma Delta Modulated Radio-over-Fiber
}

\author{
Laurens Breyne, Guy Torfs, Xin Yin, Piet Demeester, Johan Bauwelinck
}

\begin{abstract}
With the continuously increasing demand of cost effective, broadband wireless access, radio-over-fiber (RoF) starts to gain more and more momentum. Various techniques already exist, using analog (ARoF) or digitized (DRoF) radio signals over fiber. Each with their own advantages and disadvantages. By transmitting a sigma delta modulated signal over fiber (SDoF), a similar immunity to impairments as DRoF can be obtained while maintaining the low complexity of ARoF. This letter describes a detailed experimental comparison between ARoF and SDoF that quantifies the improvement in linearity and error vector magnitude (EVM) of SDoF over ARoF. The experiments were carried out using a 16QAM constellation with a baudrate from 20 to 125 Mbaud modulated on a central carrier frequency of $1 \mathrm{GHz}$. The sigma delta modulator (SDM) runs at 8 or $13.5 \mathrm{Gbps}$. A high speed VCSEL operating at $850 \mathrm{~nm}$ is used to transmit the signal over $200 \mathrm{~m}$ multimode fiber. The receiver amplifies the electrical signals and subsequently filters to recover the original RF-signal. Compared to ARoF, improvements exceeding $40 \mathrm{~dB}$ were measured on the third order intermodulation products when SDoF was employed, the EVM improves between 2.4 to $7.1 \mathrm{~dB}$.
\end{abstract}

Index Terms-Microwave Photonics, Analog Radio-Over-Fiber, Digitized Radio-Over-Fiber, Sigma-Delta-over-Fiber, Sigma Delta Modulation, Linearity, Error Vector Magnitude

\section{INTRODUCTION}

$\mathbf{O}$ VER the last years, there has been a continuously growing interest in radio-over-fiber (RoF). Especially as an enabling technology for next generation mobile communication networks, e.g. $5 \mathrm{G}$ and $60 \mathrm{GHz}$ networks [1]. The key aspects of RoF are providing a cheap and efficient way of distributing high frequency radio signals from a central office (CO) to several remote radio heads (RRHs). Several variants of RoF, each with their own distinctive advantages and disadvantages have emerged. These are discussed first.

In the most straightforward case, a digital signal is transformed to the analog domain using a digital to analog converter (DAC) and transmitted through the optical link, such that an analog radio-over-fiber (ARoF) link is obtained. An example is shown in Fig. 1a. In general, ARoF provides the

Manuscript received June 10, 2017; revised August 18, 2017; accepted September 11, 2017. Date of publication XX XX, 2017; Date of current version XX XX, 2017. This work was partly supported by the European Commission in the framework of the 2020-ICT-2014-2 project Flex5Gware (Grant agreement no. 671563) and by the iMinds IoT research program. Piet Demeester thanks the ERC for his advanced grant 695495 "ATTO: A new concept for ultra-high capacity wireless networks". Laurens Breyne has a PhD fellowship from the Special Research Fund (BOF) - Ghent University.

L. Breyne, G. Torfs, X. Yin, P. Demeester and J. Bauwelinck are with Ghent University - imec, IDLab, Department of Information Technology, 9052 Ghent, Belgium (email: Laurens.Breyne@UGent.be).

Copyright (c) 2017 IEEE. Personal use of this material is permitted. However, permission to use this material for any other purposes must be obtained from the IEEE by sending a request to pubs-permissions@ieee.org.

Digital Object Identifier XX most spectrally efficient solution with the least complex and most power efficient RRH. However, this method is prone to distortion and non-linearities at both the transmitter and receiver. Furthermore, DACs, oscillators and mixers at the transmitter consume much power.

An alternative is digitized-radio-over-fiber (DRoF), as shown in Fig. 1b. The digital signal is serialized and transmitted. A binary driver replaces the DAC at the transmitter. At the RRH, the digital stream is deserialized, transformed to the analog domain using a DAC and, if required, upconverted to recover the original analog RF signal. Transmitting digital data mitigates non-linearity issues at both the transmitter and receiver [2]. Furthermore, various standards already exists for DRoF, e.g. CPRI [3] and OBSAI [4]. For high carrier frequencies and high baudrates, the cost and power consumption of the DAC and, if implemented, the upconversion at the RRH become prohibitively large. It becomes difficult to have a fixed phase relation between several RRHs. Transmitting digitized samples instead of the analog signal itself results in a very low spectral efficiency. These disadvantages are especially troubling when many RRHs have to be supported [5].

A technique combining the advantages of ARoF and DRoF, i.e. digital communication with a simple, power efficient RRH while completely eliminating the need for high-speed, high resolution DACs is provided by sigma delta-over-fiber (SDoF) [6], [7], [8] and as shown in Fig. 1c. The power hungry DAC and the subsequent linear driver at the ARoF transmitter are replaced by a sigma delta modulator (SDM) and a binary driver. The digital signal transmitted over the link is highly immune to non-linearities, similar to DRoF. At the receiver, the original analog signal is recovered using an appropriate filter, resulting in a simple, power efficient RRH. In fact, the same RRH can be used for ARoF and SDoF. In a highly linear link, for example when using a DFB laser, SDoF and ARoF will have a comparable performance. However, when a more nonlinear VCSEL is used to decrease the power consumption, the use of SDoF will be advantageous.

In this letter, a detailed comparison between $\mathrm{ARoF}$ and SDoF is made by directly modulating a multimode VCSEL and placing an analog bandpass filter after the optical receiver. The SDM is implemented in Matlab ${ }^{\circledR}$ as a bandpass sigma delta modulator (BPSDM), explained in the next section. Thereafter, we briefly discuss the measurement setup. The linearity is assessed using third order intermodulation products (IM3), the total link quality is measured using the error vector magnitude (EVM). Various biasing points of the VCSEL, SDM sample rates and input signal amplitudes for the VCSEL and the BPSDM were considered in the comparison. 


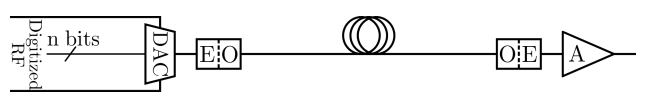

(a)

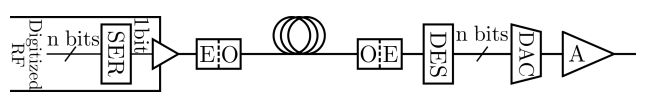

(b)

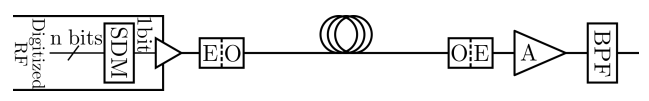

(c)

Fig. 1. Examples of an (a) ARoF, (b) DRoF and (c) SDoF link. E-O: Electrical-to-optical; O-E: Optical-to-electrical; A: Amplifier; SER: Serializer; DES: Deserialiser; SDM: Sigma Delta Modulator; BPF: Bandpass filter.

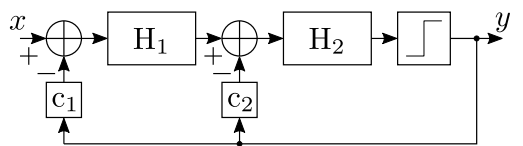

Fig. 2. Structure of the SDM employed in this paper. The combination of $H_{1}$ and $H_{2}$, both second order transfer functions with the feedback constants $c_{1}$ and $c_{2}$ yields a $4^{\text {th }}$ order SDM. The quantizer has a resolution of one bit.

\section{Sigma DELTA OVER FIBER}

The essence of sigma delta modulation consists of quantizing a signal, typically with a very low resolution, at a sample rate much higher than minimally required by the Nyquist criterion. In the frequency domain, this allows to reshape the relatively high quantization noise such that the noise in the band of interest can be decreased significantly, resulting in a very high signal to noise ratio (SNR) [9]. By bandpass filtering the reshaped spectrum afterwards, the quantization noise can be suppressed to retrieve the original signal.

The block diagram of the employed SDM is given in Fig. 2. The combination of $H_{1}$ and $H_{2}$, both second order transfer functions, with the feedback constants $c_{1}$ and $c_{2}$ yields a fourth order SDM. The one-bit quantizer is used to obtain a 2-level signal (1 or -1$)$ at the output. In a first approximation, it can be assumed that the input range is equal to the output range, thus the full scale input range is from -1 to 1 . In this work, the SDM input amplitude is expressed with respect to this full scale range. A simple analysis of this structure can be performed by replacing the quantizer with a linear approximation, i.e. omitting the quantizer and only adding (white) quantization noise [9]. The transfer function from the input $x$ to the output $y$ when the quantizer is neglected is the signal transfer function (STF). Equivalently, the transfer function from the quantization noise input to the output $y$ when the input is zero is the noise transfer function (NTF).

In our case, the input signal is a narrowband RF-signal with carrier frequency $F_{\text {center}}$, such that the STF should be a bandpassfilter with a passband that encompasses $F_{\text {center }}$. To maximize the SNR, the NTF should be a bandstopfilter centered around $F_{\text {center }}$. The transfer functions of the employed SDM, $H_{1}$ and $H_{2}$, are given by:

$$
H_{1}=\frac{1-B z^{-1}}{1-2 B z^{-1}+z^{-2}}, \quad H_{2}=\frac{\left(B-z^{-1}\right) z^{-1}}{1-2 B z^{-1}+z^{-2}}
$$

And the constant $B$ is given by:

$$
B=\cos \left(2 \pi F_{\text {center }} / F_{\text {sample }}\right)
$$

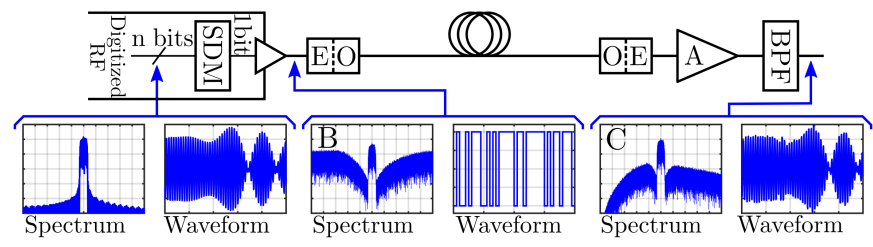

Fig. 3. The SDoF link annotated with the spectra and time domain waveforms at several points: A. the (digital) input of the SDM; B. the (electrical) output of the SDM and C. at the output of the receiver.

with $F_{\text {sample }}$ the sample frequency at which the SDM is operating. The constants $c_{1}$ and $c_{2}$ are fixed and equal to 2.5 and 3 respectively. This results in a NTF magnitude below $-40 \mathrm{~dB}$ over $200 \mathrm{MHz}$ around the $1 \mathrm{GHz}$ center frequency and a STF magnitude around $-8 \mathrm{~dB}$ over the same frequency band, assuming the sample rate is at least $8 \mathrm{Gbps}$. FPGA and ASIC implementations of sigma delta modulators running at very high rates have already been demonstrated [8].

The principle of sigma delta modulation can now be applied to RoF, see Fig. 3. In the digital domain, the digital RF signal with a spectrum shown in inset $A$ of Fig. 3 is sigma delta modulated resulting in the spectrum of inset B. As shown in the associated waveforms, a 2-level signal is obtained. This signal is transmitted and bandpass filtered at the receiver, resulting in the spectrum shown in inset $\mathrm{C}$ in Fig. 3. Thus the 2-level signal has been reshaped to the original RF-signal. Notwithstanding the higher complexity in the digital domain, this implementation allows to significantly decrease the power consumption at the transmitter since no high-speed, highresolution DAC is required and the 2-level signal allows the use of a nonlinear optical source, e.g. a VCSEL. One of the main disadvantages of SDMs is the significant power of the quantization noise with respect to the power of the signal itself. Nevertheless, this low coding efficiency can be compensated by the increased efficiency of the transmitter itself. In this letter, only the downlink is considered. Given the need for digital processing and the intention of keeping the RRH simple, SDoF is less attractive for the uplink.

\section{EXPERIMENTAL SETUP}

Before discussing the measurement results, an overview of the measurement setup is given in Fig. 4. The RF-signal is generated offline and sigma delta modulated using Matlab ${ }^{\circledR}$. The sigma delta stream is uploaded to the pulse pattern generator (PPG), capable of generating bitstreams up to $13.5 \mathrm{Gbps}$. The outputs (-1 or 1$)$ of the SDM are mapped on a 2-level signal $\left(-V_{P P G}\right.$ or $\left.V_{P P G}\right)$. The generator directly drives a $50 \mathrm{Gbps}$, $850 \mathrm{~nm}$, multimode VCSEL [10]. The measured $3 \mathrm{~dB}$ electrooptical bandwidth of the VCSEL is well above $15 \mathrm{GHz}$ for driving currents exceeding $4 \mathrm{~mA}$. For comparison, the optical link consists of 1 meter patchcord or 200 m OM4 multimode fiber. In this work, we focus on short range optical links. A $5 \mathrm{GHz}$ PIN photodetector is followed by a receiver and analog bandpass filter to detect, amplify and filter the optical signal. To protect the photodetector, $7 \mathrm{~dB}$ optical attenuation is inserted in the link. This is equivalent to $14 \mathrm{~dB}$ electrical attenuation. The conversion gain of the complete receiver (photodetector, receiver and bandpass filter) at $1 \mathrm{GHz}$ is around 


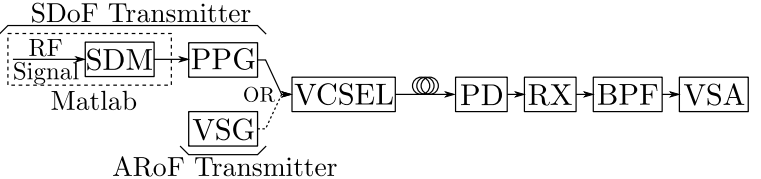

Fig. 4. Experimental setup for the SDoF and ARoF link tests. SDM: Sigma delta modulator; PPG: Pulse pattern generator; VSG: Vector signal generator; PD: Photodetector; RX: Receiver; BPF: Bandpass Filter; VSA: Vector signal analyzer.

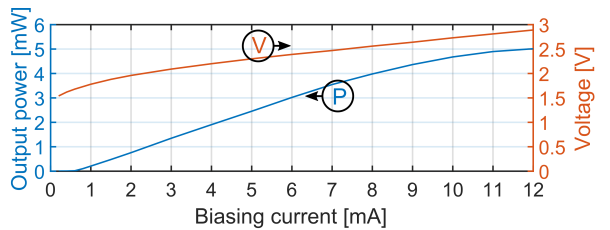

Fig. 5. Optical output power and forward voltage of the VCSEL as a function of driving current. The threshold current is $0.6 \mathrm{~mA}$ while the threshold voltage is $1.7 \mathrm{~V}$.

$220 \mathrm{~V} / \mathrm{W}$, while the bandpass $3 \mathrm{~dB}$ bandwidth is $190 \mathrm{MHz}$, the spectral noise current density around $1 \mathrm{GHz}$ is $10 \mathrm{pA} / \sqrt{\mathrm{Hz}}$. The EVM measurement is done using a vector signal analyzer (VSA). To compare ARoF and SDoF, the SDoF transmitter is replaced by a vector signal generator (VSG). The VSG has a maximal baudrate of 80 Mbaud. Note that a more linear link can be devised by using a DFB laser, which will improve ARoF performance at the cost of power consumption.

\section{EXPERIMENTAL Results AND Discussion}

First, the DC characteristics of the VCSEL are measured, since they already give a good indication of the VCSEL linearity. Both the optical output power and the forward voltage of the VCSEL are given in Fig. 5 as a function of driving current. It can be observed that the VCSEL driving current should be sufficiently large to avoid subtreshold operation [6]. On the other hand, gain compression occurs at higher currents. This is especially detrimental for ARoF. In the following experiments, the biasing points are chosen at 4 and $8 \mathrm{~mA}$. This implies that the amplitude of the PPG output should sufficiently stay below 0.5 and $0.9 \mathrm{~V}$ respectively. For ARoF, the peak power of the RF-signal should remain below 4 and $9 \mathrm{dBm}$ respectively to avoid that the VCSEL operates below its threshold.

\section{A. Linearity Comparison}

The linearity of the complete optical link, i.e. from the input of the VCSEL to the output of the complete receiver (see Fig. 4), is measured using a two-tone measurement. The center frequency is chosen at $1 \mathrm{GHz}$, the spacing between both tones equals $10 \mathrm{MHz}$. First, the power of the fundamental tone and third order intermodulation product (IM3) were measured at the receiver output when the SDoF transmitter was selected. Thereafter, the SDoF transmitter was replaced by the ARoF transmitter. The power of the ARoF transmitter was set such that the fundamental tone at the receiver output has a power similar to the power of the fundamental tone when the SDoF transmitter is used. In order to compare both results, the IM3 can be plotted as a function of the fundamental output power for both cases. Given the complex system for SDoF, i.e. digital

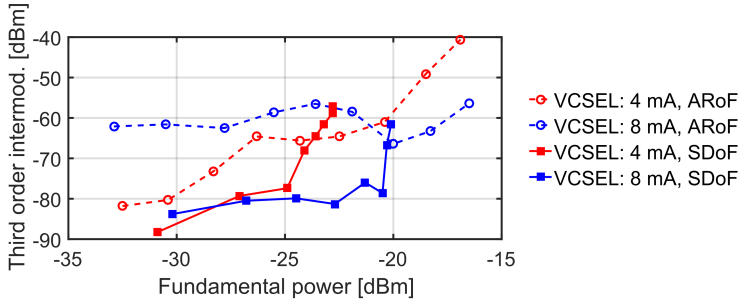

Fig. 6. Third order intermodulation as a function of the fundamental output power for ARoF and SDoF on various biasing points of the VCSEL. The PPG output voltage is swept while keeping the SDM input amplitude constant

$\mathrm{BBU}$ and analog RRH, care should be taken when interpreting the IM3 measurements. The results of this measurement are shown in Fig. 6. The input signal of the SDM has an amplitude of 1.5 , while the PPG output amplitude $\mathrm{V}_{\mathrm{PPG}}$ is swept from 0.2 to $0.9 \mathrm{~V}$. This way, only the nonlinear effects of the optical link are included. The finite length of the sigma delta modulated bitstream will increase the noise floor compared to the theoretical case. Although the input amplitude exceeds unity, due to the attenuation of the STF, no notable distortion is observed. The SDM is operating at $8 \mathrm{Gbps}$.

When the VCSEL is biased at $4 \mathrm{~mA}$, the IM3 clearly improves when SDoF is employed. For fundamental powers below $-25 \mathrm{dBm}$, the power of the IM3 tones generated using SDoF drops 7 to $14 \mathrm{~dB}$ compared to ARoF. The sudden increase in IM3 of the SDoF measurement at fundamental powers above $-25 \mathrm{dBm}$ is caused by subtreshold operation of the VCSEL. The discrepancy between SDoF and ARoF becomes even more pronounced when the VCSEL is biased at $8 \mathrm{~mA}$, since $\mathrm{ARoF}$ starts to experience the detrimental effects of gain compression, leading to an increased IM3. The $3^{\text {th }}$ order intermodulation in the SDoF case is 14 to $24 \mathrm{~dB}$ lower compared to ARoF. The sudden increase in IM3 at a fundamental power of $-21 \mathrm{dBm}$ in the $\mathrm{SDoF}$ measurement is again caused by the subthreshold operation of the VCSEL.

The same measurement can now be repeated, but the output voltage of the PPG is kept constant while the amplitude of the input signal of the SDM is swept from 0.1 to 1.5 (0.2 to 3 peak-to-peak). The third order intermodulation introduced by the SDM is now also taken into account. The resulting IM3 as a function of the fundamental power, when the VCSEL is biased at $8 \mathrm{~mA}$, is shown in the left part of Fig. 7. The PPG amplitude is set to $0.6 \mathrm{~V}$, which is sufficiently far from threshold to avoid distortion. For fundamental powers between -35 and $-25 \mathrm{dBm}$, the IM3 component is 26 to $45 \mathrm{~dB}$ lower in the SDoF case. The VCSEL causes significant compression in the ARoF case when the fundamental output power exceeds $-30 \mathrm{dBm}$. Remark that for very low input amplitudes, the SDM has an IM3-floor caused by the finite bitstream length.

In the right part of Fig. 7, the signal to interference ratio (SIR) is given. For SDoF, the SIR remains fairly constant and only starts to drop at the end, while for ARoF, a gradual decrease is observed. This is in line with earlier results [1].

\section{B. Error Vector Magnitude Comparison}

A more realistic experiment consists of transmitting an I/Q modulated RF-signal, performing demodulation and calculating the error vector magnitude (EVM). A 16QAM signal 


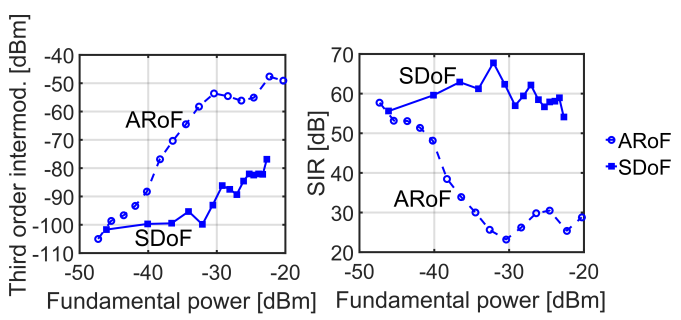

Fig. 7. Left: Third order intermodulation as a function of the fundamental output power for ARoF and SDoF with the VCSEL biased at $8 \mathrm{~mA}$. The PPG output voltage is kept constant while sweeping the SDM input amplitude. Right: The SIR as a function of the fundamental output power, derived from the data in the left figure.
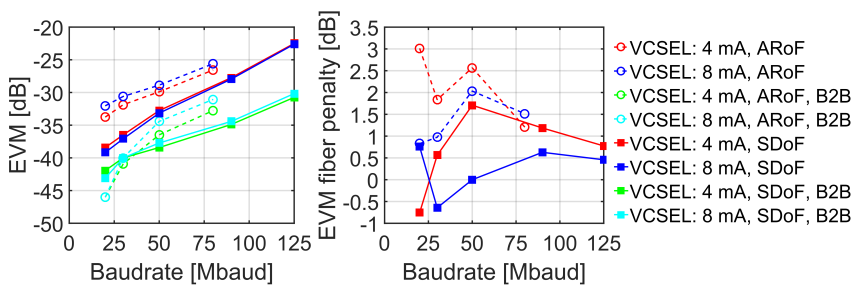

Fig. 8. Left: EVM when the PPG is directly connected to the VSA (electrical B2B) and EVM when the signal is transmitted over a link of a few meters. Right: EVM penalty when the optical link length is increased from a few meter to $200 \mathrm{~m}$ OM4 fiber.

was transmitted on a $1 \mathrm{GHz}$ carrier. The roll-off of the root raised cosine pulse was 0.35 . For every measurement, the input amplitude and output voltage swing of the SDM and PPG were optimized to minimize the EVM. The results of this measurement are shown in Fig. 8. The SDM was running at $13.5 \mathrm{Gbps}$. The electrical back-to-back (B2B) curves give the EVM when the output of the PPG is directly connected to the vector signal analyzer (VSA). In the left part of Fig. 8, the optical link length is a few meters, while the right part shows the EVM penalty when the link length is increased to $200 \mathrm{~m}$ OM4 fiber. The RF-power of the vector signal generator (VSG) is set such that the power of the RF-signal is equal to the RF-power at the output of the receiver when SDoF is used.

In the left part of Fig. 8, it is observed that the EVM improves 2.4 to $7.1 \mathrm{~dB}$ if $\mathrm{SDoF}$ is employed. Looking to the EVM of ARoF over the optical link, it is observed that the EVM increases with increasing baudrate. A higher baudrate results in a higher total noise power in the signal band and thus an increased EVM. For SDoF the increase in EVM is larger due to the bandstop shape of the quantization noise. In the theoretical case where the baudrate goes to zero, the EVM will not go to zero, since nonlinearities will cause intermodulation. For ARoF, the $8 \mathrm{~mA}$ case performs slightly worse compared to the $4 \mathrm{~mA}$ case due to the VCSEL gain compression. Thus a part of the EVM difference between ARoF and SDoF is determined by the nonlinearity. In the right part of Fig. 8, the EVM penalty between the short and longer optical link is observed. Due to the high bandwidth-distance product of OM4 fiber, only a limited EVM penalty is observed.

The effect of changing the sample rate of the SDM is shown in Fig. 9. The VCSEL was biased at $8 \mathrm{~mA}$, the SDM output voltage and input amplitude were optimized for minimal EVM, the baudrate was 100 Mbaud and the spectra were measured directly at the PPG output. For 3 Gbps, the EVM is $-32 \mathrm{~dB}$,

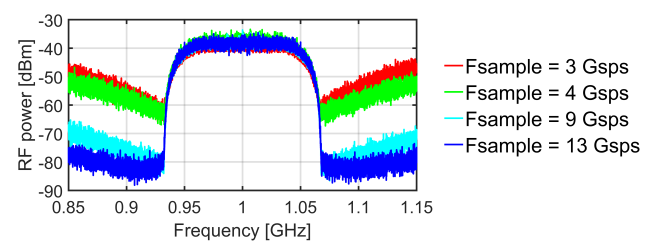

Fig. 9. Spectra at the output of the PPG for various sample rates of the SDM. SDM input amplitude: 2.5 , PPG amplitude: $0.5 \mathrm{~V}$.

at $4 \mathrm{Gbps}$, the EVM drops to $-34 \mathrm{~dB}$. At 9 and $13 \mathrm{Gbps}$, the EVM is $-34.5 \mathrm{~dB}$. Thus only for lower sample rates, the EVM experiences a mild increase. More important is the decrease in adjacent channel power (ACP) for increasing sample rates, since it is difficult to decrease the ACP using analog filtering. A low ACP is important to comply with spectral masks. At $13 \mathrm{Gbps}$, the small increase in noise around the signal is caused by sampling artifacts.

\section{CONCLUSion}

A detailed experimental comparison between ARoF and SDoF was made, based on linearity and EVM. Using a twotone measurement, the third order intermodulation product of the SDoF link was in certain cases $40 \mathrm{~dB}$ lower compared to ARoF for the same fundamental tone power. When transmitting a 16QAM signal at baudrates between 25 and 125 Mbaud modulated on a $1 \mathrm{GHz}$ carrier, EVM improvements between 2.4 to $7.1 \mathrm{~dB}$ were found. While the sigma delta modulator was running at 13.5 Gbps. Furthermore, it was shown that the sample rate can be relatively low before the EVM starts to rise. Lowering the sample rate, however, comes at the cost of an increased ACP. Overall, this letter shows that a RoF link with a cheap optical transmitter with relative low linearity (e.g. a VCSEL) benefits from employing SDoF over ARoF and DRoF due to the improved analog performance, decreased complexity and increased flexibility.

\section{REFERENCES}

[1] D. Novak et al., "Radio-over-fiber technologies for emerging wireless systems," IEEE Journal of Quantum Electronics, vol. 52, no. 1, pp. 1-11, Jan 2016.

[2] H. D. Jung, K. W. Lee, J. H. Kim, Y. H. Kwon, and J. H. Park, "Performance comparison of analog and digitized rof systems with nonlinear channel condition," IEEE Photonics Technology Letters, vol. 28, no. 6, pp. 661-664, March 2016.

[3] "Common Public Radio Interface (CPRI)," [Online]. Available: http://www.cpri.info.

[4] "Open Base Station Architecture Initiative (OBSAI)," [Online]. Available: http://www.obsai.com.

[5] A. Haddad and M. Gagnaire, "Radio-over-fiber (rof) for mobile backhauling: A technical and economic comparison between analog and digitized rof," in 2014 Int. Conf. on Optical Network Design and Modeling, May 2014, pp. 132-137.

[6] L. M. Pessoa, J. S. Tavares, D. Coelho, and H. M. Salgado, "Experimental evaluation of a digitized fiber-wireless system employing sigma delta modulation," Opt. Express, vol. 22, no. 14, pp. 17 508-17 523, Jul 2014.

[7] S. Jang, G. Jo, J. Jung, B. Park, and S. Hong, "A digitized if-overfiber transmission based on low-pass delta-sigma modulation," IEEE Photonics Technology Letters, vol. 26, no. 24, pp. 2484-2487, Dec 2014.

[8] D. Markert, X. Yu, H. Heimpel, and G. Fischer, "An all-digital, singlebit rf transmitter for massive mimo," IEEE Transactions on Circuits and Systems I: Regular Papers, vol. 64, no. 3, pp. 696-704, March 2017.

[9] R. Schreier and G. C. Temes, Understanding Delta-Sigma Data Converters, 1st ed. Hoboken, N.J.: John Wiley \& Sons, 2004.

[10] “V50-850M Datasheet," Vertically Integrated Systems, 2016. 